\title{
Dileptons and Direct Photons at SPS
}

\author{
Ruben Shahoyan
}

CERN, PH, CH-1211, Geneva 23, Switzerland

\begin{abstract}
The study of dilepton and direct photon emission was one of the main topics of the experimental program at the SPS devoted to the search of signals for QGP formation. Three generations of experiments, Helios-3, NA38/NA50, CERES and NA60 measured $e^{+} e^{-}$or $\mu^{+} \mu^{-}$production in various colliding systems and at different energies. While lepton pair production in $\mathrm{p}-\mathrm{A}$ collisions was found to be reasonably well described by the expected sources, all experiments observed in nuclear collisions an excess of the yield above the extrapolation from $\mathrm{p}-\mathrm{A}$. As a result of this joint experimental effort we have currently a large amount of information characterizing this excess: its mass spectrum over the full range from $0.2 \mathrm{GeV} / \mathrm{c}^{2}$ up to the $J / \psi$, its transverse momentum spectra including their mass dependence, its angular distributions, its dependence on collision centrality over the complete range etc. Putting together all this information leads to the conclusion that what we observe is the long-sought thermal radiation from the fireball.
\end{abstract}

\section{Introduction}

The properties of the dilepton and real photon production in relativistic heavy ion collisions constitute a significant fraction among the various signatures of the Quark-Gluon Plasma (QGP) formation. Being produced at all stages of the collision and practically lacking the final state interactions, they convey dual information. From one side, their production rate and kinematics are sensitive to the properties of the surrounding matter: dominance of the partonic or hadronic constituents, temperature, density, flow etc. In turn, the hot and dense matter is expected to affect spectral functions of the dilepton emission. Disentangling these two effects poses both interpetational and experimental problems: the "conventional" sources of the observed effects need to be understood, and the small production rates and large backgrounds require large integrated luminosity measurements.

\section{Experimental results}

The mentioned difficulties are the reason behind the scarce results on direct photons collected so far at the SPS. 15-20\% upper limits (95\% CL) on the excess over hadronic sources $\left(\pi^{0}, \eta\right.$ and $\eta^{\prime}$ decays) were set by WA80 [1] and [2] in the central S-Au collisions at $200 \mathrm{~A} \mathrm{GeV}$. The most significant result [3] is the observation of up to $20 \pm 7 \%$ excess for $p_{T}>1.5 \mathrm{GeV} / \mathrm{c}$ in $\mathrm{Pb}-\mathrm{Pb}$ collision at $158 \mathrm{~A} \mathrm{GeV}$. It is well described by theoretical models involving pQCD photons and thermal emission both from hadron gas and QGP [4], but the interpretation is ambiguous: the thermal photon emission rate is determined by the temperature and close to the transition point both phases provide similar rates. 
The situation is much more advanced in the dilepton sector. Conventionally, its mass spectrum is separated into three regions, distingished by their dominant contributions in $\mathrm{p}-\mathrm{p}$ and $\mathrm{p}-\mathrm{A}$ collisions: (i) Low Mass Region (LMR, $M<M_{\phi}$ ) where the emission is mostly determined by the leptonic decays of vector mesons (resonant part) and non-resonant contribution from Dalitz decays; (ii) Intermediate Mass Range (IMR, $M_{\phi}<M<M_{J / \psi}$ ) composed of Drell-Yan dileptons and pairs coming from uncorrelated decays of open charm (mostly $D$ and $\bar{D}$ mesons); (iii) High Mass Range (HMR, $M>M_{J / \psi}$ ) dominated by the heavy quarkonia and Drell-Yan pairs. This part of the spectrum was covered by [5] in this volume and will not be considered here.

\subsection{Excess in LMR and IMR}

With arrival of the ion beams on CERN SPS all dilepton experiments observed an enhancement of the $l^{+} l^{-}$production with respect to the extrapolation from p-A collision. Helios/3 [6] and NA38 [7] experiments reported the continuum excess in $\mu^{+} \mu^{-}$, comparing the S-W and S-U collisions at $200 \mathrm{~A} \mathrm{GeV}$ respectively with $\mathrm{p}-\mathrm{W}$ interactions at the same energy.

An important milestone was set by the CERES experiment which measured electron pairs using a system of two RICH detectors. It observed a strong $(5 . \pm 0.7$ (stat) \pm 2.(syst)) LMR excess in $e^{+} e^{-}$production in $\mathrm{S}-\mathrm{Au}$ collision [8] with respect to the "cocktail" of hadronic decays [9], well describing the $\mathrm{p}$-Be and $\mathrm{p}$-Au spectra [9]. The triggered theoretical activity, trying to describe these data, was not successful until the appearence of calculations involving in-medium $\pi \pi$ annihilation via a modified $\rho$ spectral function. The model [10], exploring the idea of the Brown-Rho scaling [11], assumed a decrease of the $\rho$ pole mass close to the chiral symmetry restoration point, while [12] predicted a brodening of the $\rho$, with significant contribution from the interactions with baryons. Unfortunately, the low statistics and insufficient $(\sim 6 \%)$ mass resolution did not allow to give preference to one of the models. The excess was later confirmed $(2.73 \pm 0.23($ stat $) \pm 0.65($ syst $) \pm 0.82($ decays $))$ in $\mathrm{Pb}-\mathrm{Au}$ interactions at $158 \mathrm{~A} \mathrm{GeV}$. Fig 1 left $)$ shows the $e^{+} e^{-}$combined 95/96 spectra together with the calculations [10] and [12].
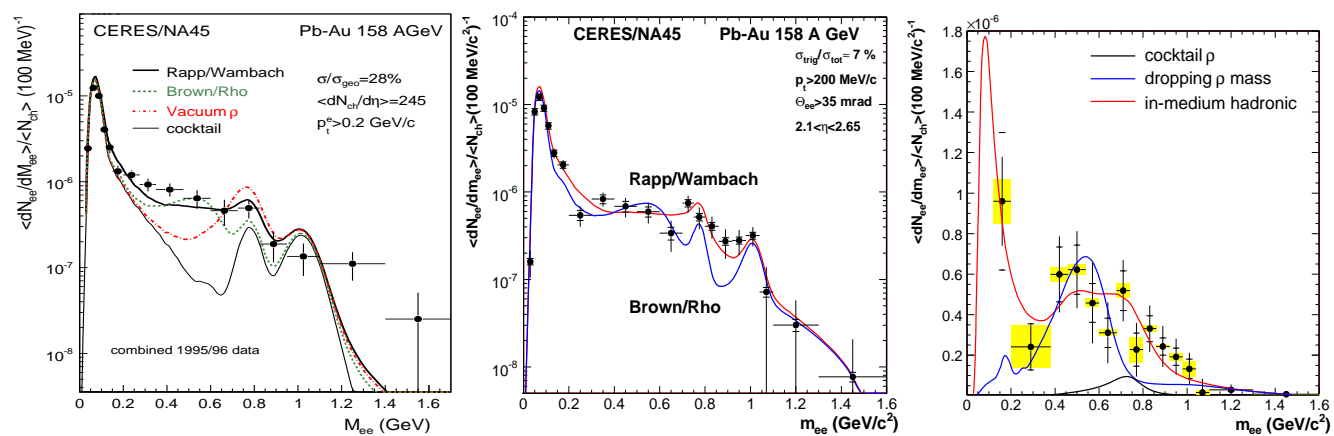

Figure 1: CERES inclusive $e^{+} e^{-}$mass spectra for $\mathrm{Pb}-\mathrm{Au}$ at $158 \mathrm{~A} \mathrm{GeV}$ compared to models [10] and [12]. (Left): combined 95/96 data [13], (Center) 2000 data,(Right) Same data after the subtraction of the hadronic "cocktail" [14].

After the CERES upgrade by the TPC in 1999 (which improved the mass resolution to 4\%), the only existing SPS dilepton measurement at $40 \mathrm{~A} \mathrm{GeV}$ was done [15]. Enhancement $5.9 \pm$ $1.5($ stat $) \pm 1.2($ syst $) \pm 1.8($ decays $)$ was reported in $\mathrm{Pb}-\mathrm{Au}$ collisions, stronger than at $158 \mathrm{GeV}$. The latter was remeasured in 2000 [14] with the result $2.6 \pm 0.3$ (stat) \pm 0.4 (syst) \pm 0.8 (decays), see Fig. 1 (center). Fig. 1 right) shows the isolated excess of $2000 \mathrm{~Pb}-\mathrm{Pb}$ data, with the hadronic decay contributions subtracted using the rates provided by the statistical model. The possible explanation of the significantly larger excess at lower energy is the higher baryonic density due to the stronger stopping. 
In the same period another important result was provided by NA50 [16]. Using the muon spectrometer inherited from NA38 and equipped with a very selective $\mu \mu$ trigger system, it observed in $\mathrm{Pb}-\mathrm{Pb}$ collisions at $158 \mathrm{~A} \mathrm{GeV}$ an IMR excess with respect to the expected Drell-Yan and open charm contributions. These latter were extrapolated from the p-A dimuon spectra at $450 \mathrm{GeV}$. Combined with the reanalyzed S-U data of the NA38, the excess showed an approximately linear rise with the number of participants, reaching a factor 2 in the central $\mathrm{Pb}-\mathrm{Pb}$ collisions. The excess, which had a kinematics resembling that of the open charm, could be accounted for [17] either by some mechanism enhancing the latter contribution in the NA50 acceptance window or by the long sought thermal dimuons [18].

The recent results are dominated by the precise and high statistics dimuon measurements of the NA60 experiment [19]. Its upgrade of the NA50 setup by a radiation tolerant silicon pixel vertex tracker (VT) [20] placed in a 2.5 Tesla dipole magnet between the target and the hadron absorber provided numerous advantages with respect to its predecessors. Particularly, by matching the muons from the spectrometer to tracks reconstructed in the VT, NA60 significantly improved the dimuon mass resolution (from $\sim 80 \mathrm{MeV} / \mathrm{c}^{2}$ to $\sim 20 \mathrm{MeV} / \mathrm{c}^{2}$ at $\omega$ mass) and was able to measure the offset of the muons wrt the production vertex with a resolution of $\sim 50 \mu \mathrm{m}$ : enough to distinguish between prompt dimuons and those coming from the open charm decays.

In 2003 NA60 has collected 230 millions dimuon triggers from In-In collisions at 158 A GeV. Data were taken with two current settings in the spectrometer magnet: $4 \mathrm{kA}$ for enhanced acceptance at low mass and $p_{T}$ and $6.5 \mathrm{kA}$ to enhance the IMR and HMR statistics.
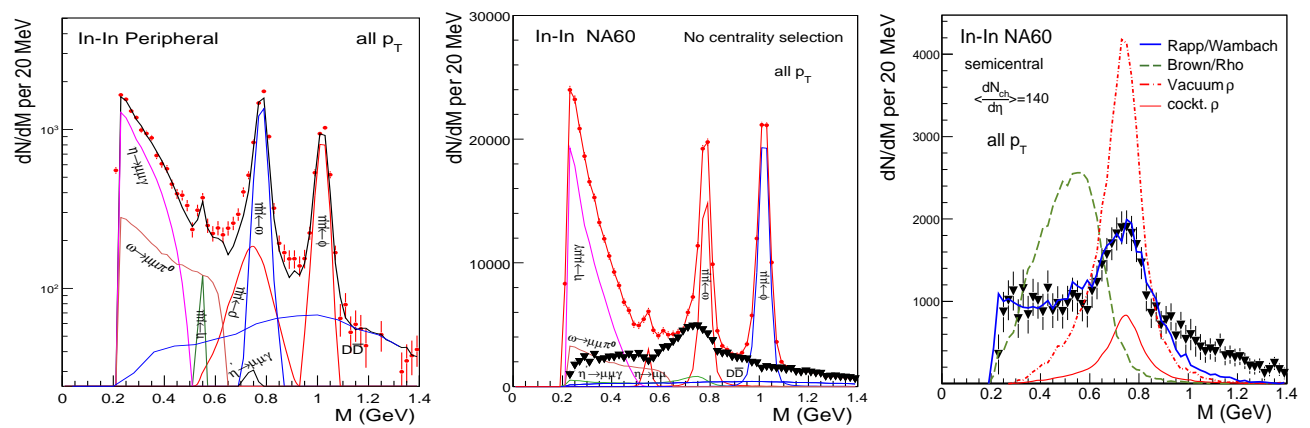

Figure 2: NA60 dimuon mass spectra measured in In-In collisions at $158 \mathrm{~A} \mathrm{GeV}$ : (Left) peripheral [21] (Center) integrated over centrality (excess is shown by triangles) [22] (Right) Isolated excess in semicentral collisions [22] compared to the Brown-Rho and Rapp-Wambach models [23]. The "cocktail" $\rho$ is not subtracted.

Fig 2l left) shows the signal dimuon mass spectrum for peripheral $\left(d N_{c h} / d \eta<30\right)$ In-In collisions [21]. It is well described by the "cocktail" of hadronic decays obtained using the GENESIS generator [9] improved and adapted for dimuons [24]. The high statistics and good mass resolution allowed direct fit of the $\eta \rightarrow \mu^{+} \mu^{-} \gamma$ and $\omega \rightarrow \mu^{+} \mu^{-} \pi^{0}$ decays form-factors [25] with the pole approximation $F=\left(1-M^{2} / \Lambda^{2}\right)^{-1}$, yielding $\Lambda^{-2}$ (in $\mathrm{GeV}^{-2}$ ) $1.95 \pm 0.17$ (stat.) \pm 0.05 (syst.) and $2.24 \pm 0.06$ (stat.) \pm 0.02 (syst.) respectively. The values agree with previous measurements by the Lepton-G experiment [26], improving their errors and confirming the strong enhancement of the $\omega$ form-factor with respect to the VMD expectation of $\Lambda^{-2}=1.68 \mathrm{GeV}^{-2}$ [27]. These measurements, together with the improved value of the $\omega \rightarrow \mu^{+} \mu^{-} \pi^{0}$ branching ratio ([1.73 \pm 0.25 (stat.) \pm 0.14 (syst.)] $10^{-4}$ ), significantly decrease the uncertainty in the hadronic decay "cocktail" below the $\omega$ in the analysis of more central collisions. Fig2 (2enter) shows the LMR mass spectrum integrated over centrality [22]. Thanks to the good mass resolution the narrow peaks of $\phi, \omega$ and $\eta$ as well as the $\eta, \eta^{\prime}$ and $\omega$ Dalitz decays can be locally subtracted 
uncovering a significant excess (shown by triangles) centered around the $\rho$ (its "cocktail" contribution is not subtracted). Fig 2 (right) compares the isolated excess for the semi-central collisions $\left(d N_{c h} / d \eta=140\right)$ [22] with the preditictions of Brown-Rho [10] and Rapp-Wambach [12] models [23]. The complete disagreement with the dropping mass and nice agreement with the broadening scenario is obvious. A similar conclusion [14], although with less statistical siginificance, is obtained by CERES from the Pb-Au (2000) data (Fig. 1 right)).
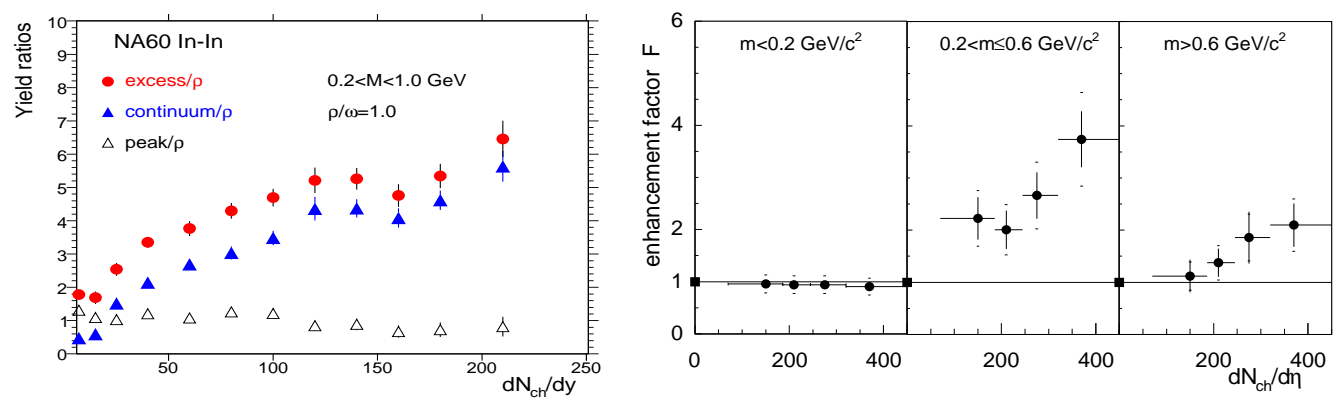

Figure 3: Evolution of the LMR excess with the multiplicity. (Left) NA60, In-In at 158 A GeV[21]. (Right) CERES, combined 95/96 Pb-Au at $158 \mathrm{GeV}$ data [13]. See text for details.

Fig. 3 (left) shows the centrality dependence of the LMR excess studied by NA60. The total "excess"(circles) is separated into continuum (filled triangles) and peak (hollow triangles) parts and related to expected "cocktail" $\rho$ contribution (assuming $\rho / \omega=1$ on the cross section level). While the peak contribution agrees with the $\rho$ produced at freeze-out, the continuum part shows a monotonic rise and broadening with centrality[21]. Such a faster than linear rise of the excess with multiplicity is compatible with emission from the annihilation process. It can be conjectured that the magnitude of the continuum excess directly measures the lifetime of the fireball in number of $\rho$ generations: so called $\rho$-clock [28]. The centrality dependence of the excess seen by CERES [13] in Pb-Au collisions, shown in Fig. 3 right) as a ratio of the excess to the total "cocktail" expectations, is in a good agreement with NA60 results.

The analysis of the NA60 IMR mass spectrum [29] in the In-In data is done by fitting the signal in the $1.16<M<2.56 \mathrm{GeV} / \mathrm{c}^{2}$ range with a superposition of the Drell-Yan and open charm contributions obtained from the Pythia 6.325 generator. The fits are done in terms of multiplicative factors for the reference cross sections. The latter are defined in the following way: for the Drell-Yan, it reproduces the cross sections measured above the $\psi^{\prime}$ by NA3 [30] and NA50 [31], while for the open charm the result of a similar fit to NA50 p-A data at $450 \mathrm{GeV}$ [16] rescaled to $158 \mathrm{GeV}$ by Pythia is used $\left(\sigma_{c \bar{c}}=8.6 \mu \mathrm{b}\right)$. Due to the insufficient high-mass DrellYan statistics, the integrated effective luminosity is extracted from the number of $J / \psi$ events and its cross section (corrected for the nuclear and anomalous suppression effects). Fig. 4 left) shows such a fit to the centrality integrated dimuon mass spectra with low current setting in the spectrometer. At this level the results are fully compatible with observations of NA50 in $\mathrm{Pb}-\mathrm{Pb}$ data [16]: while the Drell-Yan contribution agrees well with the expectations (and the data above $\psi^{\prime}$ ), a strong excess with a mass shape resembling the open charm contribution is found. The global fit to both data sets leads to the enhancement factors $1.26 \pm 0.09$ for Drell-Yan and $2.61 \pm$ 0.20 for open charm. To clarify the origin of this excess NA60 uses its excellent muon offset resolution to separate (statistically) the open charm (off-vertex decays) and prompt contributions. Fig. 4 (right) shows the dimuon "offset' 1 distribution for the mass range indicated in the left

${ }^{1}$ Defined as $\Delta_{\mu}=\left(\mathbf{d V}^{-1} \mathbf{d}^{T}\right)^{1 / 2}$ for the single muons and $\Delta_{\mu \mu}=\left[\left(\Delta_{\mu 1}+\Delta_{\mu 2}\right) / 2\right]^{1 / 2}$ for the dimuons, with $\mathbf{d}$ and $\mathbf{V}$ 
panel, fitted to the expected open charm and prompt (assumed to be Drell-Yan) contributions. The global fit to low and high current data sets leads to enhancement factor of $2.29 \pm 0.08$ and $1.16 \pm 0.16$ for the prompt and open charm samples. Hence the excess should be attributed to the prompt emission, while the open charm contribution is compatible with the extrapolation from the NA50 pA data.
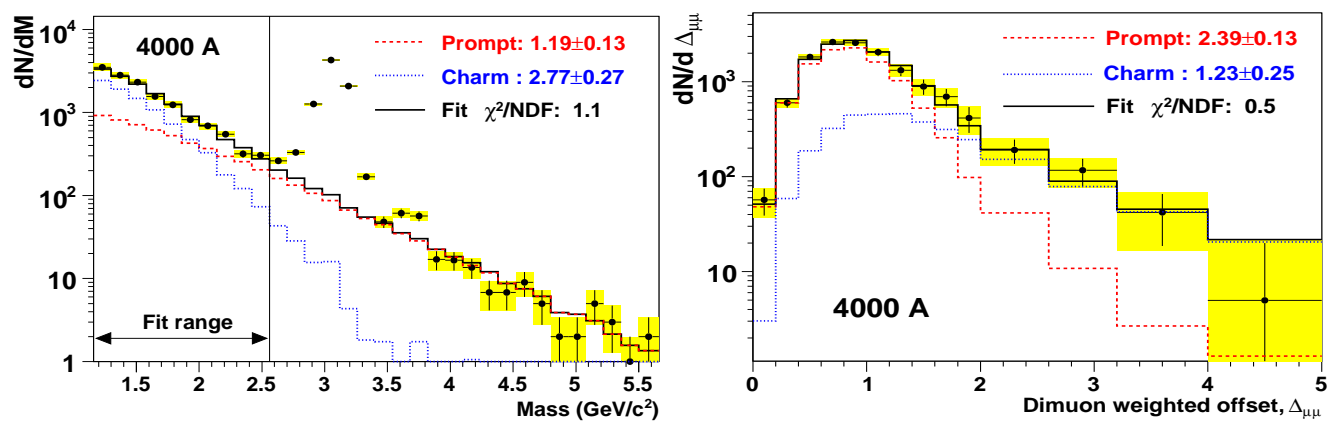

Figure 4: IMR signal dimuon distributions in In-In collisions at $158 \mathrm{GeV}$ [29]: (Left) mass spectra fitted in IMR by the superposition of Drell-Yan and open charm contributions; (Right) same for weighted offset spectra.

The statistics of the NA60 IMR spectra is not high enough to extract the open charm contribution differentially (in centrality, $M, p_{T}$ etc.). For this reason, in the differential analysis the kinematic distributions for open charm and Drell-Yan are taken from Pythia spectra, while for the dependence on centrality both are assumed to scale with the number of binary collisions extracted from the measured number of $J / \psi$ events in a given $d N_{c h} / d \eta$ bin (corrected for the suppression). The IMR excess is defined as the difference between the measured signal and the sum of these two contributions.

The dependence of the excess on the number of the collision participants shows a faster than linear scaling with the number of binary collisions (with excess/DY reaching a factor $2.3 \pm 0.7$ for the most central with $N_{\text {part }}>200$ ), but is slower than quadratic increase with the squared number of participants[29]. As in the case of the LMR excess such a behaviour is compatible with the emission from the annihilation in thermalized medium. Fig. 5 (left) summarizes the mass spectrum of the excess seen in the In-In collisions [29], corrected for the acceptance and reconstruction efficiency and normalized to the per charged particle yield.

NA60 performed also an extensive study of the $\mu \mu m_{T}$ spectra [32], summarized on Fig. 5 (right). Both hadrons and excess $m_{T}$ spectra are well described by the $d N / d m_{T}^{2} \propto \exp \left(-m_{T} / T_{\text {eff }}\right)$ form, except for some puzzling softening of the excess at masses $<1.2 \mathrm{GeV} / \mathrm{c}^{2}$ in the range of $m_{T}-M<0.2 \mathrm{GeV}$. All hadrons - $\eta, \omega, \rho$ (defined as the peak on top of the LMR excess) and $\phi$ show the rise of $T_{\text {eff }}$ with mass characteristic for the "blue-shift" due to radial flow. The deviations $T_{\rho}>T_{\phi}, T_{\omega}$ from nearly linear scaling with mass $\left(T_{\mathrm{eff}} \approx T_{0}+M<\beta>^{2}\right)$ are compatible with different freeze-out times of the corresponding hadrons due to their different coupling to the expanding medium. This is supported by a "blast-wave" analysis [33]: the $\phi$ decouples first, when the flow is not yet fully developed, while the $\rho$, whith its strong coupling to pions, freezes out last and profits from the full flow (with $T_{\text {eff }}$ reaching $\sim 300 \mathrm{MeV}$ in the most central collisions). A similar increase of $T_{\text {eff }}$ with mass is observed for the LMR excess. Since, due to the "soft point" in the equation-of-state [34] the (eventually produced) partonic phase at SPS

being the vector and corresponding covariance matrix of the transverse offset of the muon wrt. the primary vertex 
energies is not expected to develop significant flow, this suggest an emission from the thermalized hadronic expanding gas. Surprisingly, the rise of the excess $T_{\text {eff }}$ with mass changes to the flat behaviour after the sudden drop by nearly $50 \mathrm{MeV}$ at $M \sim 1 \mathrm{GeV} / \mathrm{c}^{2}$. The most plausible explanation of this effect is that the excess at these masses is dominated by thermal dimuons from a partonic source lacking significant flow.
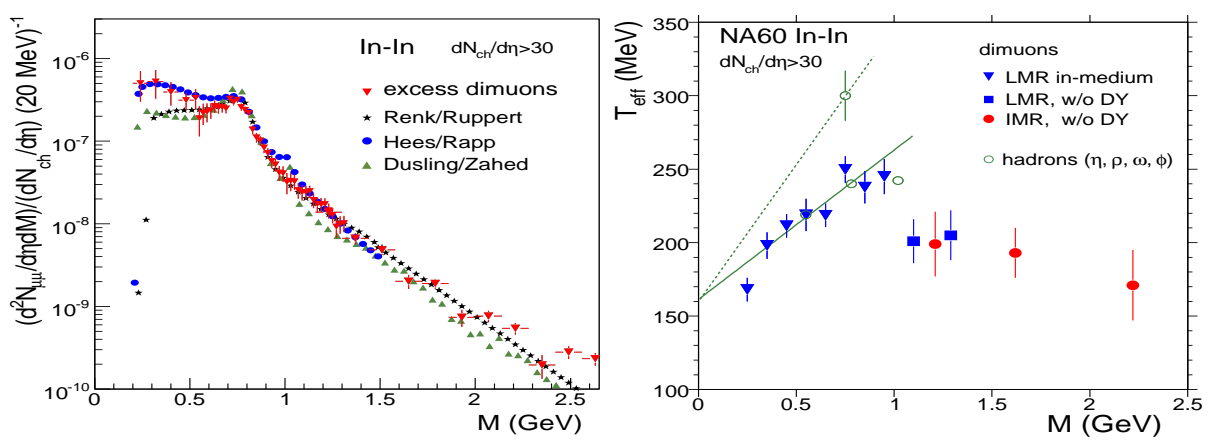

Figure 5: (Left)Acceptance corrected mass spectrum of the $\mu^{+} \mu^{-}$excess (the "coctail" $\rho$ is not subtracted) in In-In collisions [29] (Right) $\mathrm{T}_{\text {eff }}$ of excess (the $\rho$ peak contribution is subtracted) vs. dimuon mass [33] 35].

Such an interpetation is supported by the theoretical calculations. The models labeled Hees/ Rapp [36] and Renk/Rupppert [37] assume the dominant contribution in the LMR to be $\pi^{+} \pi^{-}$ annililation via modified $\rho$ spectral function ([12] and [38] respectively), while the IMR excess is defined by the combination of $4 \pi$ annihilation processes and a significant contribution from the annihilation in the partonic phase. They differ in the estimate of the fraction of the latter: [36] puts emphasis on chiral mixing via the $\pi a_{1} \rightarrow \mu^{+} \mu^{-}$process, keeping the partonic contribution within $20-60 \%$ (depending on the fireball evolution scenario), while [37] assumes $\sim 80 \%$ contribution from the partonic phase. The model Dusling/Zahed [39] uses hydrodynamic calculation with a virial expansion for the rates in the hadronic phase and $q \bar{q}$ annihilation in the partonic one. The latter contributes $60-90 \%$ to the IMR excess. All models roughly agree with the data. The differences at low masses reflect the differences in the tail of the $\rho$ spectrum ([36] with a strong effect from baryons provides the best description). A detailed comparison of these models with data in narrow $p_{T}$ bins is contained in [33].

The thermal origin of the excess is further supported by the abscence of any polarization in the excess dimuons [40, 41]. All coefficients of the $d \sigma / d \Omega \propto\left(1+\lambda \cos ^{2} \theta+\mu \sin 2 \theta \cos \phi+\frac{v}{2} \sin ^{2} \theta \cos 2 \phi\right)$ parameterization are found to be compatible with zero, which is a necessary (though not sufficient) condition of the emission from an isotropic thermalized source.

\section{2. $\omega$ and $\phi$ mesons}

Despite various predictions ([36] and references therein), no anomaly in the pole positions and widths of the $\omega$ and $\phi$ mesons was observed so far within the experimental reach of NA60. Due to the longer lifetimes, only a small fraction of these mesons produced in the medium contributes to the observed dilepton signal. For this reason any in-medium modifications will have much weaker effect on the measured $\omega$ and $\phi$ spectra than for the $\rho$. Besides that, the possible broadening of the $\omega$ would be practically inobservable since it would merge with the $\rho$ peak. Instead one could look for the deficit of low- $p_{T} \omega$ dileptons (with strongest contribution from in-medium decays) in the nominal pole position. NA60 has reported the first observation of such an effect [32] for the $\omega$. While the $p_{T}$ spectra of the $\phi$ in the whole $p_{T}$ range and of the 
$\omega$ at $p_{T}>0.8 \mathrm{GeV} / \mathrm{c}$ are perfectly thermal and agree very well with the "blast-wave" fits at all centralities, the low- $p_{T} \omega$ spectra become gradually depleted as centrality increases, with almost complete disappearence of the $\omega$ with $p_{T}<0.2 \mathrm{GeV} / \mathrm{c}$ for the most central collisions.

Significant attention was paid recently to so called $\phi$-puzzle: the disagreement between $\phi \rightarrow K^{+} K^{-}$measured by NA49 [42] and $\phi \rightarrow \mu^{+} \mu^{-}$measured by NA50 [43]. NA50, whose $\phi$ acceptance is limited by $p_{T}>1.1 \mathrm{GeV} / \mathrm{c}$ sees nearly twice the yield observed by NA49 whose statistics is limited by $p_{T}<1.6 \mathrm{GeV} / \mathrm{c}$, with a significant difference in the inverse slope parameters: $T_{\mu \mu}=234 \pm 7$ vs. $T_{K K}=305 \pm 15 \mathrm{MeV}$ in the central Pb-Pb collisions. Recent measurements by CERES [44] of $\phi$ production in central $\mathrm{Pb}-\mathrm{Au}$ collisions at $158 \mathrm{~A} \mathrm{GeV}$ both in the $K^{+} K^{-}$and $e^{+} e^{-}$channels are consistent with each other and seem to confirm the NA49 results (although with large errors on the $T_{\text {eff }}$ in $e^{+} e^{-}$). NA60 has reported similar measurements of both $K^{+} K^{-}$and $\mu^{+} \mu^{-}$channels in In-In collisions [45]. Like CERES, it finds a good agreement between the two channels, with $T_{\text {eff }}($ Fig. 6 right)) compatible with the observations of NA49 for the same number of participants, but it observes a smaller $\phi$ (Fig. 6 left)) yield per participant than NA50 and slighly stronger than both NA49 and CERES do. Taking into account that NA50 recently reanalysed its $\mathrm{Pb}-\mathrm{Pb}$ data and confirmed the previous results [46], at present, it is difficult to reconcile all of the observations into a coherent picture, albeit there is some hint for a possible physics mechanism leading to a difference in the two channels [47].
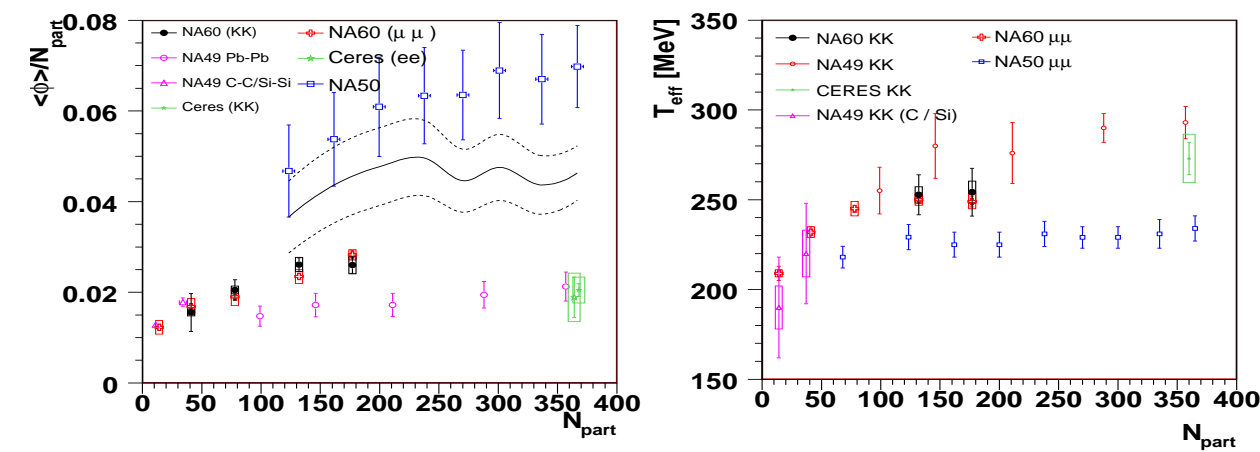

Figure 6: Mean $\phi$ multipliciy per participant (Left) and $T_{\text {eff }}$ (Right) as a function of the number of participants 45].

\section{Conclusions}

A summary of dilepton measurements at the SPS was presented. The most prominent result is the excess observed in heavy ion collisions at all masses below the $J / \psi$. Its most plausible explanation by the production of thermal dimuons is supported by the thermal-like spectra both in mass and in transverse momentum, the lack of any polarisation and by the reasonable agreement with theory. At low masses, the $l^{+} l^{-}$pairs are dominated by $\pi \pi$ annihilation via the short-lived $\rho$ meson broadened by the hot medium (the Brown-Rho scenario of a dropping $\rho$ mass is ruled out by the data). The monotonic rise of $T_{\text {eff }}$ with mass up to $M \sim 1 \mathrm{GeV} / \mathrm{c}^{2}$ with a sudden drop and then stabilization at higher masses, seen by NA60, suggests that in the IMR the dominant contribution is due to the $q \bar{q}$ anniliation in the partonic phase, when the radial flow has not yet developed. The suppression of the low- $p_{T} \omega$ mesons in the central In-In collisions hints on the first observation of its in-medium modifications. The $\phi$-puzzle: the contradiction between the $l^{+} l^{-}$and $K^{+} K^{-}$decay channels studied by the NA50 and NA49 experiments in Pb-Pb collisions is not solved despite the consistent results obtained for both channels by CERES and NA60 in $\mathrm{Pb}-\mathrm{Au}$ and In-In collisions, respectively. 


\section{Acknowledgments}

The author is grateful to the organizers of the Quark Matter 2009 conference for financial support of his participation.

\section{References}

[1] R. Albrecht et al., (WA80 Coll.), Phys. Rev. Lett. 763506 (1996).

[2] R. Baur et al., (CERES Coll.), Z. Phys. C 71571 (1996).

[3] M. M. Aggarwal et al., (WA98 Coll.), Phys. Rev. Lett. 853595 (2000).

[4] S. Turbide, R. Rapp and C. Gale, Phys. Rev. C 69014903 (2004).

[5] R. Arnaldi for NA60 Coll., Proceedings of Quark Matter 2009, this volume.

[6] A. L. Angelis et al., (Helios/3 Coll.), Eur. Phys.J C 13433 (2000).

[7] C. Lourenço et al. (NA38 Coll.), Nucl. Phys. A566 77c (1994).

[8] G. Agakichiev et al., (CERES Coll.), Phys. Rev. Lett. 751272 (1995).

[9] G. Agakichiev et al., (CERES and TAPS Coll.), Eur. Phys.J. C 4231 and 249 (1998).

[10] G. Q. Li, C. M. Ko and G. E. Brown, Phys. Rev. Lett. 754007 (1995).

[11] G. E. Brown and M. Rho, Phys. Rev. Lett. 66, 2720 (1991); Phys. Rep. 269333 (1996).

[12] G. Chanfray, R. Rapp and J. Wambach, Phys. Rev. Lett. 76368 (1996).

[13] G. Agakichiev et al., (CERES Coll.), Eur. Phys.J. C 41475 (2005).

[14] D. Adamova et al., (CERES Coll.), Phys. Lett. B 152411 (1985).

[15] D. Adamová et al., (CERES Coll.), Phys. Rev. Lett. 91042301 (2003).

[16] M. C. Abreu et al. (NA50 Coll.), Eur. Phys. J. C 14443 (2000); C. Soave, Tesi di Dottorato di Ricerca, Universita degli studi di Torino (1998).

[17] L. Capelli et al. (NA38/NA50 Coll.), Nucl. Phys. A 698 539c (2002).

[18] R. Rapp and E. Shuryak, Phys. Lett. B473 13 (2000).

[19] P. Sonderegger for NA60 Coll., Proceedings of the Quark Matter 2004 conference, J.Phys. G 30 S1101 (2004).

[20] M. Keil et al., Nucl. Instrum. Meth. A 54920 (2005).

[21] S. Damjanovic for thr NA60 Coll., Eur. Phys.J. C 49235 (2007)

[22] R. Arnaldi et al., (NA60 Coll.), Phys. Rev.Lett. 96, 162302 (2006)

[23] Calculations provided by R. Rapp in private communications to NA60.

[24] S. Damjanovic, A. De Falco and H. Wöhri, (NA60 Coll.), Internal Note 2005-1.

[25] R. Arnaldi et al., (NA60 Coll.), Phys. Lett. B 677260 (2009).

[26] R. I. Djeliadin et al., (Lepton-G Coll.), Phys. Lett. B 94548 (1980); Phys. Lett. B 102296 (1981).

[27] L. G. Landsberg et al., Phys. Reports 128301 (1985).

[28] U. Heinz, Phys. Lett. B 259162 (1991).

[29] R. Arnaldi et al., (NA60 Coll.), Eur. Phys.J. C 59607 (2009).

[30] J. Badier et al. (NA3 Coll.), Z. Phys. C 26489 (1985).

[31] M. C. Abreu et al. (NA50 Coll.), Phys. Lett. B 410337 (1997).

[32] R. Arnaldi et al., (NA60 Coll.), Eur. Phys.J. C 61711 (2009).

[33] S. Damjanovic et al. (NA60 Coll.), J. Phys. G 35104036 (2008).

[34] C. M. Hung and E. Shuryak, Phys.Rev.Lett. 754003 (1995); Phys.Rev. C 571891 (1998).

[35] R. Arnaldi et al. (NA60 Coll.), Phys. Rev. Lett. 100022302 (2008)

[36] H. van Hees and R. Rapp, Nucl.Phys. A 806339 (2008).

[37] J. Ruppert, C. Gale, T. Renk, P. Litchard and J. Kapusta, Phys.Rev.Lett. 100162301 (2008); T. Renk and J. Ruppert, Phys.Rev. C 77024907 (2008).

[38] V. L. Eletsky et al., Phys.Rev. C 64035202 (2001).

[39] K. Dusling, D. Teaney and I. Zahed, Phys.Rev. C 75024908 (2007); K. Dusling, PhD thesis, University of Stony Brook (USA), (2009), nucl-th/0901.2027/

[40] G. Usai for NA60 Coll., Proceedings of Quark Matter 2009, this volume.

[41] R. Arnaldi et al., (NA60 Coll.), Phys.Rev.Lett. 102222301 (2009)

[42] S. Afanasev et al., (NA49 Coll.), Phys. Lett. B 49159 (2000); J. Phys. G 27355 (2001).

[43] B. Alessandro et al. (NA50 Coll.), Phys. Lett. B 555147 (2003).

[44] D. Adamova et al., (CERES Coll.), Phys. REv. Lett. 96152301 (2006).

[45] A. De Falco for NA60 Coll., Proceedings of Quark Matter 2009, this volume.

[46] D. Jouan for NA50 Coll., Proceedings of Quark Matter 2008, J. Phys. G 35104163 (2008)

[47] R. Arnaldi et al. (NA60 Coll.), arXiv:0906.1102 accepted for publication in Eur.Phys. J. 